\title{
On a result of Mahler on the decimal expansions of $(n \alpha)$
}

\author{
by \\ DANiEl Berend (Beer-Sheva) and \\ Michael D. Boshernitzan (Houston, Tex.)
}

1. Introduction and the main results. It is well known that, given any irrational $\alpha$, the sequence $(n \alpha)_{n=1}^{\infty}$ is dense modulo 1 . (It is also uniformly distributed modulo 1 , but this is of no consequence here.) In particular, given any digits $a_{1}, a_{2}, \ldots, a_{k}$, there exists a positive integer $m$ for which the decimal expansion of $m \alpha$ contains this block of digits. It was proved by Mahler $[\mathrm{M}]$ that, moreover, there necessarily exists an $m$ for which the decimal expansion of $m \alpha$ contains the given block infinitely often. Mahler also established an upper bound for the minimal value $M$ of the number $m$ with that property; $M=M(k)$ depends only on the number $k$ of digits, but not on $\alpha$ :

$$
M(k)<10^{2 k+1} .
$$

Mahler's original proof is based on the geometry of numbers.

In this paper we give a shorter proof of Mahler's result (see Section 2), which at the same time yields a better upper bound:

$$
M(k)<2 \cdot 10^{k+1} .
$$

This result is best possible up to a constant factor. In fact, we show that

$$
M(k) \geq 8 \cdot\left(10^{k}-1\right) .
$$

(Actually, the factor 8 can be replaced by any real number less than 10 for sufficiently large $k$ - see Example 3.1.)

Of course, there is nothing special about the base 10. Mahler's theorem refers equally to any base $g \geq 2$, and the upper bound for $M(g, k)$ he obtains in this general case is:

$$
M(g, k)<g^{2 k+1} .
$$

(Note that even the finiteness of $M(g, k)$ is not obvious.)

Research of the second author supported in part by NSF Grant No. DMS-9003450. 
Our first result improves upon this bound. A $g$-block of length $k$ is a sequence of length $k$ with entries in $\{0,1, \ldots, g-1\}$.

THEOREM 1.1. Let $\alpha$ be an irrational, $g \geq 2$ an integer and $B$ a $g$-block of length $k$. Then there exists a positive integer $m<2 g^{k+1}$ such that the $g$-ary expansion of $m \alpha$ contains the block $B$ infinitely often.

The theorem is proved in Section 2.

Remark. As is well known, the $g$-ary expansion of almost every $\alpha$ (in the sense of the Lebesgue measure) contains every $g$-block infinitely often (and even in the "right" frequency). The theorem thus relates mainly to numbers $\alpha$ which are "badly behaved" in base $g$.

It is easy to see (Proposition 3.1) that

$$
M(g, k) \geq g^{k}-1 .
$$

Thus the gap between our upper and lower bounds is just by a factor of $2 g$, which is constant (for fixed $g$ ). In Section 3 we shall discuss improvements upon this lower bound. Our lower bounds depend on the arithmetic nature of $g$ (i.e., its factorization into a product of primes), and may hint that there is no simple formula for $M(g, k)$.

The density modulo 1 of the sequence $(n \alpha)$ is but a special case of a result which asserts that, given any polynomial $P$ with real coefficients, at least one of which (besides the constant term) is irrational, the sequence $P(n)$ is dense modulo 1. (More well-known is Weyl's even stronger result by which this sequence is uniformly distributed modulo 1 [W].) It turns out that Mahler's result is true in this more general setting as well.

Theorem 1.2. Let $g \geq 2$ be an integer and $P \in \mathbb{R}[x]$ a polynomial with at least one irrational coefficient besides the constant term. Then for each finite $g$-block there exists a positive integer $m$ such that $B$ appears infinitely often in the g-ary expansion of $P(m)$.

Remark. It can be shown (although this does not follow from the considerations of this paper) that there exists an effective upper bound, in terms of $g$, the length of $B$ and the degree of $P$, on the least $m$ satisfying the conclusion of the theorem.

EXAMPLE 1.1. There are numerous sequences in which one can find, given any $g$-block, an element whose $g$-ary expansion contains the block infinitely often. Such are the sequences $(\ln n)$ (consider numbers $n$ of the form $2^{m}$ and use Mahler's result), $(\ln \ln n)$ (take $n$ 's of the form $2^{2^{m}}$ and use Theorem 1.2 for linear polynomials) and $\left(n^{\theta}\right)$ for $\theta$ positive rational non-integer (if $\theta=p / q$ take $n$ 's of the form $2 m^{q}$ and use Theorem 1.2). On the other hand, we do not know whether the sequences $(\ln \ln \ln n)$ and $\left(n^{\theta}\right)$ with irrational $\theta$ share this property. More generally, we note that the 
question of infinite repetitions of blocks is usually harder than the question of density mod 1 which holds for the above sequences. For a large class of regularly growing sequences (defined by certain formulae or recurrences), the questions of density and of uniform distribution modulo 1 can be resolved by means of simple tests $[\mathrm{B}]$, but we doubt that such criteria can be formulated for infinite repetition problems.

We note in conclusion that our approach was influenced by an idea due to Furstenberg who, employing a certain result of Glasner [G], provided a very short proof of the finiteness of $M(g, k)$ (see [AP, Cor. 7.2]).

2. The improved upper bound. Let $\mathbb{T}=\mathbb{R} / \mathbb{Z}$ be the circle group. $A$ set $E \subseteq \mathbb{T}$ is $\varepsilon$-dense (or, alternatively, forms an $\varepsilon$-net) in $\mathbb{T}$ if every interval of length $\varepsilon$ meets $E$.

It is obvious that $M(g, k)$ cannot be divisible by $g$, so that proving a weak inequality in Theorem 1.1 is equivalent to proving the strict inequality.

Proof of Theorem 1.1. Denote by $E$ the set of all limit points in $\mathbb{T}$ of the sequence $\left\{g^{n} \alpha: n \geq 0\right\}$. We distinguish between several (somewhat overlapping) cases:

Case I: $E$ contains a rational point $r=p / q$ (where $(p, q)=1$ ) with $g^{k}<q \leq g^{k+1}$. In this case the set $\{0, r, 2 r, \ldots,(q-1) r\}$ forms a $\frac{1}{g^{k}}$-net in $\mathbb{T}$. Hence the $g$-ary expansion of some $m r, 1 \leq m<q$, starts with $0 . B$, and not all of the following digits are 0 , neither are they all $g-1$. It follows that the $g$-ary expansion of $m \alpha$ contains the block $B$ infinitely often. (Note that in this case we could have replaced the upper bound $2 g^{k+1}$ by $g^{k+1}$.)

Case II: $0 \in E$. Replacing $\alpha$ by $-\alpha$ if necessary, we may assume that the $g$-ary expansion of $\alpha$ contains arbitrarily large blocks consisting of 0 . Take a sequence $\left(n_{j}\right)$ of positive integers such that the $g$-ary expansion of $g^{n_{j}} \alpha$ starts with the block $0^{j}$, but that of $g^{n_{j}-1} \alpha$ does not start with 0 . For each fixed positive integer $d$, consider the sequence $g^{n_{j}-d} \alpha$ (which is well-defined for sufficiently large $j$ ). Replacing $\left(n_{j}\right)$ by a subsequence thereof, we may assume that each of these sequences converges in $\mathbb{T}$, say $g^{n_{j}-d} \alpha \rightarrow r_{d}=p_{d} / q_{d}$ (where $\left(p_{d}, q_{d}\right)=1$ ). Obviously, for each $d$ we have $q_{d}<q_{d+1} \leq g q_{d}$. Hence $g^{k}<q_{d} \leq g^{k+1}$ for an appropriate choice of $d$. Since $r_{d} \in E$ for each $d$, this yields a reduction to the preceding case.

Case III: $E$ contains a rational point $r=p / q$ (where $(p, q)=1$ ) with $q \leq g^{k+1}$. Carrying out the construction of Case II, with $\alpha$ replaced by $q \alpha$, we find rationals $s_{d}$ in $q E$ with finite $g$-ary expansion and corresponding rationals $r_{d}=p_{d} / q_{d}$ in $E$ such that $g^{n_{j}-d} \alpha \rightarrow r_{d}$ and $q s_{d}=r_{d}$ for each $d$. As in the preceding case we have $q_{d}<q_{d+1} \leq g q_{d}$, and since $q_{0} \leq g^{k+1}$ there exists an $r_{d}$ whose denominator is in the range $\left(g^{k}, g^{k+1}\right]$, bringing us again back to Case I.

Case IV: $E$ contains no rational point $r=p / q$ with $q \leq 2 g^{k}$. We first 
claim that a point $\beta \in E$ and a rational $r=p / q$ can be found such that:

(a) $|\beta-p / q|<1 /\left(2 g^{k+1} q\right)$.

(b) $2 g^{k}<q$.

(c) $q \leq 2 g^{k+1}$.

(d) $(p, q)=1$.

In fact, starting with any $\beta_{0} \in E$, we can find a rational $r_{0}=p_{0} / q_{0}$ such that conditions (a), (c) and (d) are satisfied with $\beta$ and $r$ replaced by $\beta_{0}$ and $r_{0}$, respectively. Choose inductively points $\beta_{i} \in E, i=0,1,2, \ldots$ with $g \beta_{i+1}=\beta_{i}$ for each $i$. Next choose rationals $r_{i}=p_{i} / q_{i}, i=1,2, \ldots$ (in reduced form) with $g r_{i+1}=r_{i}$ and $\left|\beta_{i}-r_{i}\right|=g^{-i}\left|\beta_{0}-r_{0}\right|$ for each $i$. Clearly, $q_{i} \leq q_{i+1} \leq g q_{i}$ for each $i$. If $q_{i}=q \leq 2 g^{k}$ for all sufficiently large $i$, then some rational $r=p / q$ appears infinitely often in the sequence $\left(r_{i}\right)$, in which case $r \in E$, contradicting our assumption. Consequently, $q_{i} \rightarrow \infty$, whence for a suitable $i$ the rational $r_{i}$ satisfies conditions (a)-(d).

Now the set $\{r, 2 r, \ldots, q r\}$ forms a $\frac{1}{2 g^{k}}$-net in $\mathbb{T}$. Since $|m \beta-m r|<$ $1 /\left(2 g^{k}\right)$ for $1 \leq m \leq q$, the set $\{\beta, 2 \beta, \ldots, q \beta\}$ forms a $\frac{1}{g^{k}}$-net in $\mathbb{T}$. We conclude as in Case I.

This completes the proof.

3. Lower bounds. In this section we shall discuss the question of lower bounds on $M(g, k)$. A simple observation is

Proposition 3.1. $M(g, k) \geq g^{k}-1$ for every $g \geq 2, k \geq 1$.

In fact, considering a number of the form $\alpha=\sum_{j=1}^{\infty} g^{-n_{j}}$, where $n_{j+1}-$ $n_{j} \rightarrow \infty$, and the block $B$ consists of $k$ consecutive $(g-1)$ 's, we easily see that the $g$-ary expansion of $m \alpha$ will not contain $B$ infinitely often for any $m<g^{k}-1$.

The bound provided by Proposition 3.1 may be equal exactly to $M(g, k)$. This is the case, for example, for $g=2, k=1$ and for $g=3, k=1$. It is, however, usually possible to improve on this lower bound, as we first see for composite $g$.

TheOREM 3.1. Let a be a proper divisor of $g$. Then

$$
M(g, k) \geq a\left(g^{k}-1\right), \quad k \geq 1 .
$$

Taking $a=1$ we obtain Proposition 3.1. Of course, the best result is obtained in general by selecting $a$ as the maximal proper divisor of $g$. Thus Theorem 3.1 improves Proposition 3.1 unless $g$ is a prime.

The proof of Theorem 3.1 is almost the same as that of Proposition 3.1, except that we choose the "bad number" this time as 


$$
\alpha=\frac{g}{a} \sum_{j=1}^{\infty} g^{-n_{j}} .
$$

The least multiple of $g / a$ containing the block consisting of $k$ consecutive $(g-1)$ 's is the number

$$
g^{k+1}-g=\frac{g}{a} \cdot a\left(g^{k}-1\right) .
$$

Consequently, the least positive integer $m$ for which $m \alpha$ contains the block $B$ infinitely often is $a\left(g^{k}-1\right)$, which proves the theorem.

Even more can be said if $g$ is not a prime power.

THEOREM 3.2. If $g$ is not a prime power, then for every $\varepsilon>0$ there exists a positive integer $K=K(\varepsilon)$ such that

$$
M(g, k) \geq(1-\varepsilon) g^{k+1}, \quad k \geq K .
$$

Proof. Let $p$ be a prime divisor of $g$. Since $g$ is not a prime power, $\log p / \log g$ is irrational. Therefore one can find positive integers $l$ and $r$ such that $g^{l}<p^{r}<(1+\varepsilon) g^{l}$. We first claim that the $g$-ary expansion of no positive multiple of $p^{r}$ contains the block $B$, consisting of $r-l$ consecutive $(g-1)$ 's, within its $r$ lowest digits. In fact, if this were possible, then by multiplying this multiple of $p^{r}$ by an appropriate power of $g$, we would get a number of the form $m p^{r}$ whose block of $r$ lowest digits starts with the block $B$. Since $g^{r}$ is divisible by $p^{r}$, we can find such a number with exactly $r$ digits. But then for this number $m p^{r}$ we have

$$
g^{r}-g^{l} \leq m p^{r}<g^{r} .
$$

As all three numbers involved in the inequality are multiples of $p^{r}$, this is inconsistent with the fact that $p^{r}>g^{l}$. Thus the minimal $m p^{r}$ containing a block consisting of $k \geq r-l$ consecutive $(g-1)$ 's is at least

$$
\sum_{i=l+1}^{l+k}(g-1) g^{i}=g^{l+1}\left(g^{k}-1\right) \text {. }
$$

Now set

$$
\alpha=\left(\frac{p}{g}\right)^{r} \sum_{j=1}^{\infty} g^{-n_{j}},
$$

where $n_{j+1}-n_{j} \rightarrow \infty$. The foregoing discussion implies that the smallest $m$ for which $m \alpha$ contains the block consisting of $k(\geq r-l)$ consecutive $(g-1)$ 's infinitely often is at least

$$
\frac{g^{l+1}\left(g^{k}-1\right)}{p^{r}}>\left(1-\frac{\varepsilon}{2}\right)\left(g^{k+1}-g\right)>(1-\varepsilon) g^{k+1}
$$

for sufficiently large $k$. This completes the proof. 
EXAMPLE 3.1. For $g=10$, taking $p=2, l=0, r=1$ in the proof we see that $M(10, k) \geq 5 \cdot\left(10^{k}-1\right)$ for $k \geq 1$. Taking $p=5, l=2, r=3$, we obtain a better result, namely $M(10, k) \geq 8 \cdot\left(10^{k}-1\right)$ for $k \geq 1$. With $p=2, l=3, r=10$ we get $M(10, k) \geq 9.765 \cdot\left(10^{k}-1\right)$ for $k \geq 7$.

We do not know whether it is true in general that $M(g, k)<g^{k+1}$. However, except for the cases $g=2$ and $g=3$, mentioned earlier, we never have $M(g, 1)=g-1$. In view of Theorem 3.1 we have to prove this assertion only for prime $g$. The following theorem includes this case.

Theorem 3.3. Let $g \geq 5$ be an odd integer. Then

$$
M(g, 1) \geq \frac{3}{2}(g-1) .
$$

Pr o of. Take

$$
\alpha=\frac{1}{2}+\sum_{j=1}^{\infty} g^{-n_{j}},
$$

where $n_{j+1}-n_{j} \rightarrow \infty$. One easily writes down the $g$-ary expansion of $\alpha$ and of multiples $m \alpha$. It is easily checked that if $g \equiv 1(\bmod 4)$ then the digit $(g-3) / 2$ appears at most finitely many times in the expansion of $m \alpha$ for every $m<\frac{3}{2}(g-1)$. The same is true for the digit $g-2$ if $g \equiv 3(\bmod 4)$. This proves the theorem.

4. The polynomial Mahler theorem. In this section we prove Theorem 1.2.

Define (for the purposes of this section) the complexity of a polynomial $P(x)=a_{0}+a_{1} x+\ldots+a_{d} x^{d}$ with real coefficients as the least common denominator of the numbers $a_{1}, \ldots, a_{d}$ if they are all rational and as $\infty$ otherwise.

Lemma 4.1. Given $\varepsilon>0$ and a positive integer $d$, there exists a positive integer $M$ such that for every polynomial $P$ of degree $d$ with complexity at least $M$, the set $\{P(n): n \in \mathbb{N}\}$ is $\varepsilon$-dense modulo 1 .

Proof. If $P$ is of infinite complexity, then the sequence $(P(n))_{n=1}^{\infty}$ is uniformly distributed modulo 1 , and in particular dense modulo 1. Suppose therefore that the coefficients $a_{1}, \ldots, a_{d}$ of $P$ are all rational, and let $Q$ be the complexity of $P$. Let $x_{n}=\{P(n)\}$ be the fractional part of $P(n), n=$ $1, \ldots, Q$. We ought to show that the set $\left\{x_{n}: 1 \leq n \leq Q\right\}$ is $\varepsilon$-dense in $[0,1]$ if $Q$ is large enough. We shall prove, moreover, that even the discrepancy $D_{Q}=D_{Q}\left(x_{1}, \ldots, x_{Q}\right)$ must be small as $Q$ becomes large. Indeed, according to LeVeque's Inequality (see, for example, [KN, Ch. 1, Th. 2.4]) we have

$$
D_{Q} \leq\left(\frac{6}{\pi^{2}} \sum_{h=1}^{\infty} \frac{1}{h^{2}}\left|\frac{1}{Q} S(h, Q, P)\right|^{2}\right)^{1 / 3},
$$


where

$$
S(h, Q, P)=\sum_{n=1}^{Q} \exp (2 \pi i h P(n)) .
$$

Setting $h^{\prime}=h /(h, Q)$ and $Q^{\prime}=Q /(h, Q)$, and employing some well-known estimates of exponential sums involving a rational polynomial $[\mathrm{C}],[\mathrm{S}]$ (which are, up to a multiplicative constant, best possible; Hua's original estimates [H, Ch. 7, Th. 10.1] would suit our purposes as well), we obtain

$$
|S(h, Q, P)|=(h, Q) \cdot\left|S\left(h^{\prime}, Q^{\prime}, P\right)\right| \leq h C_{1}(d) Q^{\prime 1-1 / d},
$$

where $C_{1}(d)$ depends only on $d$. Thus

$$
D_{Q} \leq\left(C_{2}(d) \sum_{h=1}^{\infty} \frac{1}{h^{2}}\left(\frac{Q}{h}\right)^{-2 / d}\right)^{1 / 3} \leq C_{3}(d) Q^{-1 /(2 d)} .
$$

Consequently, if $Q$ is sufficiently large, then $D_{Q}<\varepsilon$, which completes the proof.

Proof of Theorem 1.2. Let $B$ be a $g$-block of an arbitrary length $k$. Write:

$$
P(x)=a_{0}+a_{1} x+\ldots+a_{d} x^{d} .
$$

Let $a_{l}(1 \leq l \leq d)$ be an irrational coefficient of $P$. Let $\varepsilon=1 / g^{k+2}$. Take $M$ as in Lemma 4.1. One easily verifies that the set of limit points modulo 1 of the sequence $\left(g^{n} a_{l}\right)_{n=1}^{\infty}$ is infinite, whence there exists a sequence $\left(n_{j}\right)$ such that $g^{n_{j}} a_{l} \rightarrow b_{l}(\bmod 1)$ where $b_{l}$ is either irrational or is a rational number with denominator at least $M$. Replacing $\left(n_{j}\right)$ by a subsequence thereof, we may assume that each of the subsequences $\left(g^{n_{j}} a_{i}\right), 1 \leq i \leq d$, converges modulo 1 , say $g^{n_{j}} a_{i} \rightarrow b_{i}(\bmod 1)$. Consider the polynomial

$$
P_{0}(x)=b_{0}+b_{1} x+\ldots+b_{d} x^{d} .
$$

By Lemma 4.1 we can find a positive integer $m$ such that the $g$-ary expansion of the number $P_{0}(m)$ modulo 1 starts with the block $B 01$. But then the number $P(m)$ contains the block $B$ infinitely often in its expansion. This proves the theorem.

\section{References}

[AP] N. Alon and Y. Peres, Uniform dilations, J. Geom. Funct. Anal. 2 (1992), 1-28.

[B] M. Boshernitzan, Uniform distribution and Hardy fields, J. Analyse Math., to appear.

[C] J. R. Chen, On Professor Hua's estimate of exponential sums, Sci. Sinica 20 (1977), 711-719.

[G] S. Glasner, Almost periodic sets and measures on the torus, Israel J. Math. 32 (1979), 161-172. 
[H] L. K. Hua, Introduction to Number Theory, Springer, New York, 1982.

[KN] L. Kuipers and H. Niederreiter, Uniform Distribution of Sequences, Wiley, New York, 1974.

[M] K. Mahler, Arithmetical properties of the digits of the multiples of an irrational number, Bull. Austral. Math. Soc. 8 (1973), 191-203.

[S] S. B. Stečkin, An estimate of a complete rational trigonometric sum, Analytic Number Theory, Mathematical Analysis and their Applications, Trudy Mat. Inst. Steklov. 143 (1977), 188-207, 211; English transl.: Proc. Steklov Inst. Math. (1980), 201-220.

[W] H. Weyl, Über die Gleichverteilung von Zahlen mod. Eins, Math. Ann. 77 (1916), 313-352.

DEPARTMENT OF MATHEMATICS

AND COMPUTER SCIENCE

BEN-GURION UNIVERSITY

BEER-SHEVA 84105

ISRAEL
DEPARTMENT OF MATHEMATICS

RICE UNIVERSITY

HOUSTON, TEXAS 77251

U.S.A.

Received on 10.8.1992
(2292) 